\title{
A Significant Fluorescent Aptamer Sensor Based on Carbon Dots and Graphene Oxide for Highly Selective Detection of Progesterone
}

Hanyue Cui

Shanghai University

Huan Lu

Shanghai University

Li Li

Shanghai University

Ya-ping Ding ( $\nabla$ wdingyp@sina.com )

Shanghai university https://orcid.org/0000-0001-5434-8932

Yao Fu

Tianjin University

\section{Research Article}

Keywords: carbon dots, fluorescence, progesterone, aptamer, graphene oxide

Posted Date: November 30th, 2021

DOI: https://doi.org/10.21203/rs.3.rs-1055914/v1

License: (a) (i) This work is licensed under a Creative Commons Attribution 4.0 International License. Read Full License

Version of Record: A version of this preprint was published at Journal of Fluorescence on February 4th, 2022. See the published version at https://doi.org/10.1007/s10895-022-02896-4. 


\section{Abstract}

In this paper, a fluorescent aptamer sensor was constructed based on the carbon dots and graphene oxide. This sensor combines the excellent fluorescence performance of carbon dots with the high specificity of aptamers to detect progesterone with high sensitivity and selectivity. In the absence of progesterone, the carbon dots-aptamer system and graphene oxide form a fluorescence resonance energy transfer process(FRET), which quenches the fluorescence of the carbon dots. When progesterone is added, the aptamer specifically binds to progesterone, resulting the fluorescence of the carbon dots is recovered. At optimal conditions, the fluorescence intensity recovered by the carbon dots has a linear relationship with the concentration of progesterone in the range of $0.1-120 \mathrm{nM}$, and the detection limit is $3.3 \times 10-11 \mathrm{M}$. Besides, the sensor has satisfactory detection results of progesterone in milk, indicating that this method has a enormous potential for application in food safety.

\section{Introduction}

Progesterone $\left(P_{4}\right)$ is a kind of natural steroid, which is mostly secreted by corpus luteum[1, 2]. It plays an important role in reproductive tissue, menstrual cycle regulation, fetal growth and early pregnancy[2]. Nowadays, many clinical studies use the amount of $\mathrm{P}_{4}$ to measure the hormone level in the body[3]. However, when the amount of $\mathrm{P}_{4}$ is too high, it will cause weight gain, breast discomfort[4], mental depression, acne and other side effect[5]. Epidemiological studies have confirmed that the imbalance of progesterone levels has an impact on the incidence of breast and cervical cancer[6]. As many scholars have studied progesterone and other hormones, they have found that not only the hormones in the body, but also the female hormones in food can also have an impact on human health. Long-term consumption of foods containing progesterone, even low-concentration foods, will interfere with the normal function of human body secretion and immune system[7]. Especially milk, milk contains a considerable amount of estrogen, and progesterone is concentrated in fat[8]. When the amount of $\mathrm{P}_{4}$ in milk is too high, it will lead to breast cancer and other diseases[9]. Therefore, the detection of $\mathrm{P}_{4}$ content is of great practical significance. At present, the main detection methods of $\mathrm{P}_{4}$ are high performance liquid chromatography[10], fluorescence spectrophotometry[11], electrochemical method and so on[12, 13]. Most of these methods need expensive instruments, complex pretreatment process, cumbersome analysis process and time-consuming, so they are not suitable for large-scale field detection. However, because of the advantages of simple operation, high sensitivity and low cost, fluorescence spectrophotometry has caught the attention of researchers.

On the basis of fluorescence spectrophotometry, fluorescent sensors based on CDs have attracted more and more attention due to their fast and simple operation, excellent fluorescence performance, less harsh storage conditions and low toxicity. Carbon dots (CDs) are carbon nanoparticles with particle size less than $10 \mathrm{~nm}$, which can stably emit light[14]. At present, CDs have been used in the fields of biological imaging[15], biosensor[16], ion molecule detection[17], information anti-counterfeiting storage and so on[18]. Trapiella et al. prepared CdSe/ZnS quantum dots as a fluorescent probe to detect progesterone in 
milk[19]. Based on the toxicity of semiconductor quantum dots, and the relatively complex synthesis method. Cao et al. developed an "on-off" fluorescence sensor based on CDs to detect progesterone, which reflects the advantages of carbon dots[20], but the sensitivity and selectivity of this method are not high enough. Therefore, how to design a high-sensitivity and high-selectivity fluorescence sensor based on carbon dots to detect progesterone needs further exploration.

According to the literature, aptamers have the advantages of high specificity, high affinity, wide target range, easy modification, short preparation time, high stability and low toxicity[21, 22]. Therefore, we tried to combine carbon dots with aptamers to improve the sensitivity and selectivity of the sensor. Aptamer is a small oligonucleotide sequence or short polypeptide that can specifically recognize the target obtained by systematic evolution of ligands by exponential enrichment (SELEX) technology[23-25]. As a new type of biosensor identification element, it can be applied to the detection of food[26], drugs[27], viruses and other aspects[28], and also have a broad application in the field of biological imaging[29]. This work combines carbon dots with aptamers, that is, combines the excellent fluorescence performance and low toxicity of carbon dots with the advantages of aptamers for specific detection of the detection substance, and constructs a simple and convenient fluorescence aptamer sensor.

Therefore, this paper constructed a fluorescent aptamer sensor based on carbon dots and graphene oxide to detect $\mathrm{P}_{4}$. Among them, graphene oxide acts as a quencher to form an excellent donor-acceptor pair in the fluorescence resonance energy transfer (FRET) process with carbon dots. Energy transfers from the surface of the carbon dots to graphene oxide, resulting in fluorescence quenching of the carbon dots. When $\mathrm{P}_{4}$ is present, it specifically binds to the aptamer on the surface of the carbon dots, and the carbon dots fall off from the surface of graphene oxide, causing the fluorescence of the carbon dots to recover. Experiments show that the sensor has many advantages such as easy operation, high selectivity, high sensitivity, wide detection range, and low detection limit.

\section{Experimental Section}

\subsection{Materials and Apparatus}

Apparatus: RF-5301PC Fluorescence Spectrophotometer (Shimadzu, Japan), UV-2501PC UV Visible Spectrophotometer (Shimadzu, Japan), electronic analytical balance (Shanghai Precision Instrument Factory), ultrasonic cleaning instrument (Kunshan Ultrasonic Instrument Co., Ltd.), pH meter (Shanghai Leici instrument factory), beaker $(25 \mathrm{~mL}, 50 \mathrm{~mL}, 100 \mathrm{~mL}), 25 \mathrm{ml}$ brown volumetric flask, stirrer, pipette (10 $\mu \mathrm{L}, 200 \mu \mathrm{L}, 1000 \mu \mathrm{L})$, JEOLJEM-200CX Transmission Electron Microscope(Hitachi, Japan), AVATAR 370 Fourier Transform Infrared Spectrometer[Nicoli, USA囚, vacuum drying oven (Shanghai Yiheng Technology Co., Ltd.), autoclave.

Materials: Citric acid was purchased from Jiangsu Qiangsheng functional Chemistry Co., Ltd, ethylenediamine, 1-(3-dimethylaminopropyl)-3-ethylcarbodiimide(EDC), N-hydroxysuccinimide (NHS), 
potassium dihydrogen phosphate, potassium dihydrogen phosphate and ascorbic acid were purchased from Sinopharm Chemical Reagent Co., Ltd, progesterone aptamers:

GATTAACATTAGCCCACCGCCCACC was purchased from Shanghai Qingke biological, L-cysteine was purchased from Shanghai Blue season biological Co., Ltd, graphene oxide, progesterone, l-epinephrine, glutathione, $\mathrm{KCl}, \mathrm{NaCl}, \mathrm{MnCl}_{2} \cdot 4 \mathrm{H}_{2} \mathrm{O}, \mathrm{CdCl}_{2}$, dopamine, estrone, estradiol, acetonitrile and anhydrous sodium sulfate were purchased from Aladdin Reagent Co., Ltd, ultra pure water.

\subsection{Synthesis of the CDs}

The carbon dots was prepared by one-step hydrothermal method according to the reports in the literature[30], ultrapure water was used as the reaction solvent. First, $1.2 \mathrm{~g}$ citric acid was weighed and poured into $20 \mathrm{~mL}$ ultra pure water, then $600 \mu \mathrm{L}$ ethylenediamine was transferred into the beaker with a pipette gun. The mixed solution was ultrasonically stirred for 30 minutes until citric acid was dissolved and the solution was evenly mixed. The mixed solution was transferred to $30 \mathrm{~mL}$ PTFE autoclave, heated at $200{ }^{\circ} \mathrm{C}$ for 4 hours and cooled down to ambient temperature. Finally, the carbon dots solution was centrifuged and filtered to remove impurities, and the purified carbon dots was stored at $4{ }^{\circ} \mathrm{C}$ for further use.

\subsection{Synthesis of CDs and progesterone aptamer conjugates}

The synthesis of the conjugates of carbon dots and progesterone aptamers is through covalent coupling, that is, the process of aptamers binding to the surface of carbon dots through covalent bonds[31].First, weigh $30 \mu \mathrm{L}, 1 \mathrm{mg} / \mu \mathrm{L}$ EDC powder and $30 \mu \mathrm{L}, 0.25 \mathrm{mg} / \mu \mathrm{L}$ NHS particles in a beaker, add $1 \mathrm{~mL}$ PBS buffer solution with $\mathrm{pH}=7.4$, and then add $100 \mu \mathrm{L} C D$ s solution. The mixed solution is ultrasonically stirred for 30 min until the solution is evenly mixed. Next, $20 \mu \mathrm{L}, 10 \mu \mathrm{M}$ progesterone aptamer was added to $10 \mu \mathrm{L}$ mixed solution, and ultrasonic stirring was performed for 2 hours.

\subsection{Detection of progesterone}

Fluorescence quenching: $50 \mu \mathrm{L}, 100 \mu \mathrm{g} / \mathrm{mL}$ graphene oxide solution was added into the mixed solution of carbon dots and progesterone aptamer to quench the fluorescence of carbon dots. After stirring and ultrasonic for $1 \mathrm{~h}$, the mixture was diluted to $25 \mathrm{~mL}$ with PBS solution of $\mathrm{pH}=7.4$ and transferred to a brown volumetric flask for fluorescence detection. The fluorescence intensity measured was $\mathrm{F}_{0}$.

Fluorescence recovery: $50 \mu \mathrm{L}$ progesterone with different concentrations was added into the solution of carbon dots - aptamer - graphene oxide to recover the fluorescence. After stirring and ultrasonic treatment for $1 \mathrm{~h}$, the mixture was diluted to $25 \mathrm{~mL}$ with PBS solution of $\mathrm{pH}=7.4$ and transferred to a brown volumetric flask for fluorescence detection. The measured fluorescence intensity was $F$.

\subsection{Pretreatment of actual sample milk}

According to the previous literature, the actual sample pretreatment process is as follow[32]: weigh $2 \mathrm{~mL}$ fresh milk purchased from a supermarket in Shanghai and put it into test tube. Add $4 \mathrm{~mL}$ of acetonitrile to the test tube and sonicate for $10 \mathrm{~min}$. Then, add $5 \mathrm{~g}$ of anhydrous sodium sulfate and stir for $30 \mathrm{~s}$. Let it 
stand for $10 \mathrm{~min}$, the mixed solution was quickly centrifuged at $10000 \mathrm{rpm}$ for $5 \mathrm{~min}$, and the liquid supernatant was gathered for detection of actual samples.

\section{Results And Disscussion}

\subsection{Characteristics of CDs}

Firstly, the morphology of CDs was characterized by transmission electron microscope(TEM). As shown in Fig. 1, the carbon dots are nearly spherical particles, and they are uniformly dispersed in ultrapure water, the diameters of CDs were centered at 2.5-4.5 nm.

Then, the optical properties of CDs were studied. As shown in Fig. 2a, CDs has an obvious symmetrical strong UV absorption peak at $350 \mathrm{~nm}$, which represents the $\pi-\pi$ * transition of C=C. There is a weak UV absorption peak at $240 \mathrm{~nm}$, which represents the $n-\pi *$ transition of $C=0$ [33]. Under the excitation of $355 \mathrm{~nm}$, there is a strong emission peak at $450 \mathrm{~nm}$. Both UV absorption and fluorescence emission spectra show that the prepared carbon dots have excellent optical properties. It can be seen from Fig. $2 \mathrm{~b}$, the fluorescence emission spectra of the carbon dots does not change with the change of the excitation wavelength. When the excitation wavelength is increased from $350 \mathrm{~nm}$ to $410 \mathrm{~nm}$, the emission wavelength is kept at $450 \mathrm{~nm}$, only the emission intensity decreases, and there is no red shift. When the excitation wavelength is $355 \mathrm{~nm}$, the emission intensity of carbon dots is the strongest.

Next, the surface functional groups were characterized by FT-IR. We can see from Fig. 3, the broad absorption peak near $3480 \mathrm{~cm}^{-1}$ corresponds to the stretching vibration of $\mathrm{O}-\mathrm{H}$ functional group; the absorption peak near $1643 \mathrm{~cm}^{-1}$ corresponds to the stretching vibration of $\mathrm{C}=\mathrm{O}$ functional group; and the absorption peak near $1556 \mathrm{~cm}^{-1}$ corresponds to the in-plane bending vibration of $\mathrm{N}-\mathrm{H}$ functional group.

\subsection{Discussion on detection mechanism}

We compared the effect of fluorescence quenching and fluorescence recovery with and without aptamer. As shown in Figure 4a, when CDs and GO exist, the fluorescence of CDS can be effectively quenched with or without the addition of aptamers, because aptamers do not work in the quenching process, but the FRET process between CDs and GO. In the process of fluorescence resonance energy transfer, $\mathrm{CDs}$ is the donator and GO is the receptor. The basic condition to realize the FRET process is that the emission spectrum of the donator overlaps the absorption spectrum of the acceptor[34]. As shown in Figure 5, the emission spectra of CDs overlaps with the excitation spectra of GO in a large area, and the fluorescence emission spectra of CDs overlaps with the UV absorption spectra of GO in a small area. This phenomenon fits the conditions for the formation of FRET. In order to further prove the role of GO in the experiment, we also compared the effects of fluorescence quenching and fluorescence recovery with and without GO. As shown in Figure 4c, when GO is not added, the aptamer cannot quench the fluorescence of $\mathrm{CDs}$, so progesterone cannot be analyzed and detected effectively. 
However, when progesterone is present, the difference between the detection effect of adding and not adding aptamer is very obvious. When the aptamer was added, the specific binding of aptamer to the target led to the release of CDs and the recovery of fluorescence; When aptamer was not added, CDs could not be separated from GO, and CDs could not be released, resulting in the fluorescence could not be recovered. It shows that aptamers are very important in the whole experiment. Figure $4 \mathrm{~d}$ can visually show the detection mechanism of the experiment: (a) the blue fluorescence of CDs-aptamer under UV light; (b) the blue fluorescence of CDs-aptamer-GO under UV light is almost absent; (c) the blue fluorescence of CDs-aptamer-GO-P 4 under UV light.

\subsection{Optimization of experimental conditions}

We studied the $\mathrm{pH}$ value of the reaction, the amount of $\mathrm{GO}$, the volume of $\mathrm{CDs}$ and the volume of aptamer to explore the optimal reaction conditions. Because carbon dots is sensitive to $\mathrm{pH}$ value, we chose $\mathrm{pH}$ value between 5.5 and 8.5 to study the influence of progesterone on fluorescence intensity. As shown in Fig. $6 a, F-F_{0}$ increases when $\mathrm{pH}$ increases from 5.5 to 7.4. This is because the carbon dots will self assemble into larger particles rapidly under too acidic conditions, the oxygen-containing groups on the surface of the CDs will be oxidized slowly, and the fluorescence intensity of the CDs will be quenched, resulting in the decrease of $\mathrm{F}$. F- $\mathrm{F}_{0}$ decreases when the $\mathrm{pH}$ value increases from 7.4 to 8.5. The reason is that when the CDs is too alkaline, the structural tautomerism of the CDs occurs rapidly, the speed of deoxidation reaction slows down, and the fluorescence intensity of the CDs will be quenched, resulting in the decrease of $\mathrm{F}[35]$. Therefore, we chose a neutral environment, that is, PBS buffer solution with pH 7.4.

Next, we studied the influence of the amount of $G O$ on the fluorescence intensity $F_{0}$ of CDs after fluorescence quenching. It can be seen from Fig. $6 \mathrm{~b}$ that when the amount of GO increases from 20 to $100 \mu \mathrm{g} / \mathrm{mL}$, the quenching degree increases greatly; When the amount of GO increases from $100 \mu \mathrm{g} /$ $\mathrm{mL}$ to $200 \mu \mathrm{g} / \mathrm{ml}$, the quenching degree first decreases and then tends to be stable. This is because when the amount of GO is too small, the distance between GO and CDs is too far, and it is not easy to carry FRET process. Therefore, we choose the amount of GO when the quenching degree is the largest, that is $100 \mu \mathrm{g} / \mathrm{mL}$.

Then, we studied the influence of the volume of $\mathrm{CDs}$ on the fluorescence intensity difference value $\left(\mathrm{F}-\mathrm{F}_{0}\right)$ before and after adding progesterone. As shown in Fig. $6 \mathrm{c}$ that when the volume of CDs increases from 5 to $18 \mu \mathrm{L}$, the fluorescence intensity first increases and then decreases, and reaches the peak at $10 \mu \mathrm{L}$. The reason is that when the volume of CDs is too small, the fluorescence intensity of CDs-aptamer conjugate is low, which leads to the low fluorescence intensity after adding progesterone. When the volume of CDs exceeds the optimal value, excessive CDs will entangle or fold with the aptamer, and it is not easy to covalently couple with the aptamer. After the release of CDs, less progesterone can specifically bind with the aptamer, resulting in the reduction of the recovered fluorescence intensity. Therefore, we choose $10 \mu \mathrm{L}$ as the optimal volume of CDs. 
Finally, we studied the influence of the volume of aptamer on the fluorescence intensity difference value $\left(\mathrm{F}-\mathrm{F}_{0}\right)$ before and after adding progesterone. It can be seen from Fig. $6 \mathrm{~d}$ that when the volume of aptamer 5 increases to $35 \mu \mathrm{L}$, the fluorescence intensity first increases and then decreases, and reaches the peak at $20 \mu \mathrm{L}$. This is due to the fact that when the volume of aptamer is too small, CDs are covalently coupled with too few aptamers, and less progesterone can specifically bind with aptamers after the release of $\mathrm{CDs}$, resulting in lower fluorescence intensity of recovery. When the volume of the aptamer is too large, the long chain structure of the aptamer may intercept the fluorescence site of the fluorescence sensor, resulting in the low fluorescence intensity of the system. Consequently, we choose $20 \mu \mathrm{L}$ as the optimal volume of aptamer.

\subsection{Detection of progesterone}

Under the optimal experimental conditions, different concentrations of progesterone (0.1-120 nM) were added into CDs-aptamer-GO solution and diluted to $25 \mathrm{~mL}$ in brown volumetric flask for detection. As shown in Fig. 7a, when the concentration of progesterone increased from 0.1 to $120 \mathrm{nM}$, the fluorescence intensity of CDs aptamer recovered, and the degree of recovery gradually increased. Fig. $7 \mathrm{~b}$ shows the linear relationship between the recovered fluorescence intensity $\mathrm{F}$ and progesterone concentration $\mathrm{C}$ in the range of $0.1-120 \mathrm{nM}$. The linear equation is $\mathrm{F}=1.36728\left[\mathrm{C}_{\text {progesterone }}\right]+217.48259 \mathrm{nM}$, the correlation coefficient $R^{2}$ is 0.99768 , and the detection limit LOD is $3.3 \times 10^{-11} \mathrm{M}$. Table 1 compares the fluorescence sensor constructed for this work with other reported sensors for progesterone detection. The linear range of progesterone detection was $0.1-120 \mathrm{nM}$, and the detection limit was $3.3 \times 10^{-11} \mathrm{M}$. Compared with other work, our fluorescent sensor has a wider detection range and lower detection limit. Moreover, the simple synthesis method of carbon dots was combined with the characteristic that aptamers could specifically bind to the analytes. The sensor has the merits of convenience, high sensitivity, and can be used for the detection of progesterone.

Table 1

Comparison with other reported methods for the detection of progesterone

\begin{tabular}{|llll|}
\hline Methods & Linear range(nM) & LOD(nM) & Ref. \\
\hline Electrochemical & $0.5-180$ & 0.17 & {$[36]$} \\
\hline Fluorescence & $0-2 \times 10^{5}$ & 10.25 & {$[20]$} \\
\hline Fluorescence & $0.95-46.11$ & 0.32 & {$[19]$} \\
\hline FO-SPR & $3.18-31.8$ & 1.59 & {$[37]$} \\
\hline SWV & $0.25-22.26$ & 0.25 & {$[38]$} \\
\hline Fluorescence & $0.1-120$ & 0.033 & This work \\
\hline
\end{tabular}




\subsection{Interference Analysis}

Selectivity is a vital criterion to measure the performance of sensors. The most important characteristic of aptamers is that they can specifically identify with the detected substance. Therefore, in this work, we choose to use aptamer and carbon dots covalent coupling to detect progesterone, for the purpose of improve the selectivity of the sensor. With the purpose of evaluating the anti-interference ability of the constructed fluorescent sensor, we measured the interference of many potential substances to the sensor. We measured the difference $\mathrm{F}-\mathrm{F}_{0}$ between the fluorescence intensity of the system quenched and recovered before and after the addition of $120 \mathrm{nM}$ progesterone and $3 \times 120 \mathrm{nM}$ potential interfering substances. Interfering substances include amino acids and peptides (L-cysteine, glutathione), hormones (L-adrenaline, estrone, estradiol) and other substances (ascorbic acid, $\mathrm{KCl}, \mathrm{NaCl}, \mathrm{MnCl}_{2}, \mathrm{CdCl}_{2}$, dopamine). As shown in the Fig. 8 , the value of $\mathrm{F}-\mathrm{F}_{0}$ is very small after adding the interfering substance, which means that the interfering substance hardly interferes with the sensor, indicating that our sensor has good selectivity.

\subsection{Detection of progesterone in real samples}

With the purpose of verifying the practicability of the constructed fluorescence sensor, we used standard addition method to detect the concentration of progesterone in real milk samples. Under the best experimental conditions, we added the known concentration of progesterone standard solution into the milk. As shown in Table 2, the actual concentration, recovery and relative standard deviation of progesterone can be obtained. The recovery was in the range of $97.2-101.2 \%$, and the relative standard deviation was $1.81 \%$. It can be concluded that our fluorescent sensor has a more accurate detection of progesterone in practical application.

Table 2

Determination of different amounts of progesterone in Real Samples

\begin{tabular}{|c|c|c|c|c|c|}
\hline Sample & $\operatorname{Added}\left(\mathrm{mol} \cdot \mathrm{L}^{-1}\right)$ & Found $\left(\mathrm{mol} \cdot \mathrm{L}^{-1}\right)$ & Recovery(\%) & $M(\%)$ & $\mathrm{RSD}(\%)$ \\
\hline Milk 1 & $5.0 \times 10^{-9}$ & $4.86 \times 10^{-9}$ & 97.2 & 99.8 & 1.81 \\
\hline 2 & $6.0 \times 10^{-8}$ & $6.05 \times 10^{-9}$ & 100.8 & & \\
\hline 3 & $8.0 \times 10^{-8}$ & $8.02 \times 10^{-9}$ & 100.2 & & \\
\hline 4 & $1.0 \times 10^{-7}$ & $1.02 \times 10^{-7}$ & 101.2 & & \\
\hline
\end{tabular}

\section{Conclusion}

In conclusion, we synthesized CDs by a simple hydrothermal method, covalently coupled CDs with progesterone aptamer, and constructed a fluorescent aptamer sensor. The sensor combines the low toxicity of carbon dots and the excellent affinity of aptamers to achieve high sensitivity and high 
selectivity for the detection of progesterone. The construction method is simple and convenient, and conforms to the concept of environmental friendliness in green chemistry. Under the best experimental conditions, the detection range is $0.1-120 \mathrm{nM}$, and the detection limit is $3.3 \times 10^{-11} \mathrm{M}$. The fluorescence aptamer sensor also has good accuracy and recovery (97.2-101.2\%) in the detection of actual mik samples, which verifies its potential in practical application.

\section{Declarations}

\section{Acknowledgements(Funding)}

The authors thank the financial support by the National Nature Science Foundation of China (No. 21671132).

\section{Declaration of Interest Statement}

The authors declare that they have no known competing financial interests or personal relationships that could have appeared to influence the work reported in this paper.

\section{Ethics declarations}

The authors declare that they have read the Ethical Responsibilities of Authors carefully, the authors meets its requirements.

\section{Consent to participate}

The authors declare that they consent to participate.

\section{Consent for publication}

The authors declare that they consent to publication.

\section{Availability of data and material}

Unpublished data

\section{Code availability}

Not applicable

\section{Authors' contributions}

The first author HYC performed the design, operation and data processing of the experiment, and wrote the manuscript. HL carried out the assistance of the experiment and the inspection and modification of the manuscript. YF completed the inspection and modification of the manuscript. All authors read and approved the final manuscript. 


\section{References}

1. Ynsa, M.D., et al., Effect of hormone replacement therapy on the elemental contents of uterine tissue. Biological Trace Element Research, 2004. 101(1): p. 37-46.

2. Lishko, P.V., I.L. Botchkina, and Y. Kirichok, Progesterone activates the principal Ca2+ channel of human sperm. Nature, 2011. 471(7338): p. 387-+.

3. Ricanyova, J., et al., Molecularly imprinted adsorbents for preconcentration and isolation of progesterone and testosterone by solid phase extraction combined with HPLC. Adsorption-Journal of the International Adsorption Society, 2010. 16(4-5): p. 473-483.

4. Sherwin, B.B., Progestogens used in menopause - Side effects, mood and qualify of life. Journal of Reproductive Medicine, 1999. 44(2): p. 227-232.

5. Greendale, G.A., et al., Symptom relief and side effects of postmenopausal hormones: Results from the postmenopausal estrogen/progestin interventions trial. Obstetrics and Gynecology, 1998. 92(6): p. $982-988$.

6. Key, T.J., et al., Circulating sex hormones and breast cancer risk factors in postmenopausal women: reanalysis of 13 studies. British Journal of Cancer, 2011. 105(5): p. 709-722.

7. Andersson, A.M. and N.E. Skakkebaek, Exposure to exogenous estrogens in food: possible impact on human development and health. European Journal of Endocrinology, 1999. 140(6): p. 477-485.

8. Roelofs, J.B., et al., Relationship between progesterone concentrations in milk and blood and time of ovulation in dairy cattle. Animal Reproduction Science, 2006. 91(3-4): p. 337-343.

9. Farlow, D.W., X. Xu, and T.D. Veenstra, Consumption of Cow's Milk and Possible Risk of Breast Cancer. Breast Care, 2010. 5(1): p. 44-46.

10. Decheng, S., et al., Trace analysis of progesterone and 21 progestins in milk by ultra-performance liquid chromatography coupled with high-field quadrupole-orbitrap high-resolution mass spectrometry. Food Chem, 2021. 361: p. 130115.

11. Tschmelak, J., N. Kappel, and G. Gauglitz, TIRF-based biosensor for sensitive detection of progesterone in milk based on ultra-sensitive progesterone detection in water. Anal Bioanal Chem, 2005. 382(8): p. 1895-903.

12. Contreras Jimenez, G., et al., Aptamer-based label-free impedimetric biosensor for detection of progesterone. Anal Chem, 2015. 87(2): p. 1075-82.

13. Kreuzer, M.P., et al., Development of Electrochemical Immunosensor for Progesterone Analysis in Milk. Analytical Letters, 2007. 37(5): p. 943-956.

14. Bourlinos, A.B., et al., Photoluminescent carbogenic dots. Chemistry of Materials, 2008. 20(14): p. 4539-4541.

15. Sun, Y.-P., et al., Quantum-sized carbon dots for bright and colorful photoluminescence. Journal of the American Chemical Society, 2006. 128(24): p. 7756-7757.

16. Shen, P. and Y. Xia, Synthesis-Modification Integration: One-Step Fabrication of Boronic Acid Functionalized Carbon Dots for Fluorescent Blood Sugar Sensing. Analytical Chemistry, 2014. 86(11): 
p. 5323-5329.

17. Zhang, Y.-f., H. Maimaiti, and B. Zhang, Preparation of cellulose-based fluorescent carbon nanoparticles and their application in trace detection of Pb(II). Rsc Advances, 2017. 7(5): p. $2842-$ 2850.

18. Jiang, K., et al., Triple-Mode Emission of Carbon Dots: Applications for Advanced Anti-Counterfeiting. Angewandte Chemie-International Edition, 2016. 55(25): p. 7231-7235.

19. Trapiella-Alfonso, L., et al., Development of a quantum dot-based fluorescent immunoassay for progesterone determination in bovine milk. Biosensors \& Bioelectronics, 2011. 26(12): p. 4753-4759.

20. Cao, L., et al., Yellow-emissive carbon dots for "off-and-on" fluorescent detection of progesterone. Materials Letters, 2020. 271.

21. $\mathrm{Ma}, \mathrm{H}$. , et al., Nucleic acid aptamers in cancer research, diagnosis and therapy. Chemical Society Reviews, 2015. 44(5): p. 1240-1256.

22. Mairal, T., et al., Aptamers: molecular tools for analytical applications. Analytical and Bioanalytical Chemistry, 2008. 390(4): p. 989-1007.

23. Ellington, A.D. and J.W. Szostak, In vitro selection of RNA molecules that bind specific ligands. Nature, 1990. 346(6287): p. 818-22.

24. Li, J., et al., Hairpin fluorescence DNA probe for real-time monitoring of DNA methylation. Analytical Chemistry, 2007. 79(3): p. 1050-1056.

25. Tuerk, C. and L. Gold, Systematic evolution of ligands by exponential enrichment: RNA ligands to bacteriophage T4 DNA polymerase. Science (New York, N.Y.), 1990. 249(4968): p. 505-10.

26. Siriangkhawut, W., et al., Stripping voltammetric determination of trace cadmium and lead in Thai organic unpolished rice after ultrasound-assisted digestion. Journal of Food Composition and Analysis, 2017. 59: p. 145-152.

27. Yao, D., et al., Resonance light scattering determination of trace bisphenol $A$ with signal amplification by aptamer-nanogold catalysis. Luminescence, 2014. 29(5): p. 516-521.

28. Li, P., et al., Development and characterization of aptamer-based enzyme-linked apta-sorbent assay for the detection of Singapore grouper iridovirus infection. Journal of Applied Microbiology, 2016. 121(3): p. 634-643.

29. Wu, X., et al., DNA Aptamer Selected against Pancreatic Ductal Adenocarcinoma for in vivo Imaging and Clinical Tissue Recognition. Theranostics, 2015. 5(9): p. 985-994.

30. Zhu, S., et al., Highly photoluminescent carbon dots for multicolor patterning, sensors, and bioimaging. Angew Chem Int Ed Engl, 2013. 52(14): p. 3953-7.

31. Chu, B.C., G.M. Wahl, and L.E. Orgel, Derivatization of unprotected polynucleotides. Nucleic acids research, 1983. 11(18): p. 6513-29.

32. Gao, F., et al., Comparison of standard addition and conventional isotope dilution mass spectrometry for the quantification of endogenous progesterone in milk. Accreditation and Quality Assurance, 2016. 21(6): p. 395-401. 
33. Dong, Y., et al., Carbon-based dots co-doped with nitrogen and sulfur for high quantum yield and excitation-independent emission. Angew Chem Int Ed Engl, 2013. 52(30): p. 7800-4.

34. Dong, H., et al., Fluorescence Resonance Energy Transfer between Quantum Dots and Graphene Oxide for Sensing Biomolecules. Analytical Chemistry, 2010. 82(13): p. 5511-5517.

35. Xu, Z.-Q., et al., Mechanistic studies on the reversible photophysical properties of carbon nanodots at different $\mathrm{pH}$. Colloids and Surfaces B-Biointerfaces, 2015. 130: p. 207-214.

36. Zhu, Y., et al., An antibody-aptamer sandwich cathodic photoelectrochemical biosensor for the detection of progesterone. Biosensors \& Bioelectronics, 2020. 160.

37. Daems, D., et al., Competitive inhibition assay for the detection of progesterone in dairy milk using a fiber optic SPR biosensor. Analytica Chimica Acta, 2017. 950: p. 1-6.

38. Jimena Monerris, M., et al., Integrated electrochemical irnmunosensor with gold nanoparticles for the determination of progesterone. Sensors and Actuators B-Chemical, 2012. 166: p. 586-592.

\section{Figures}

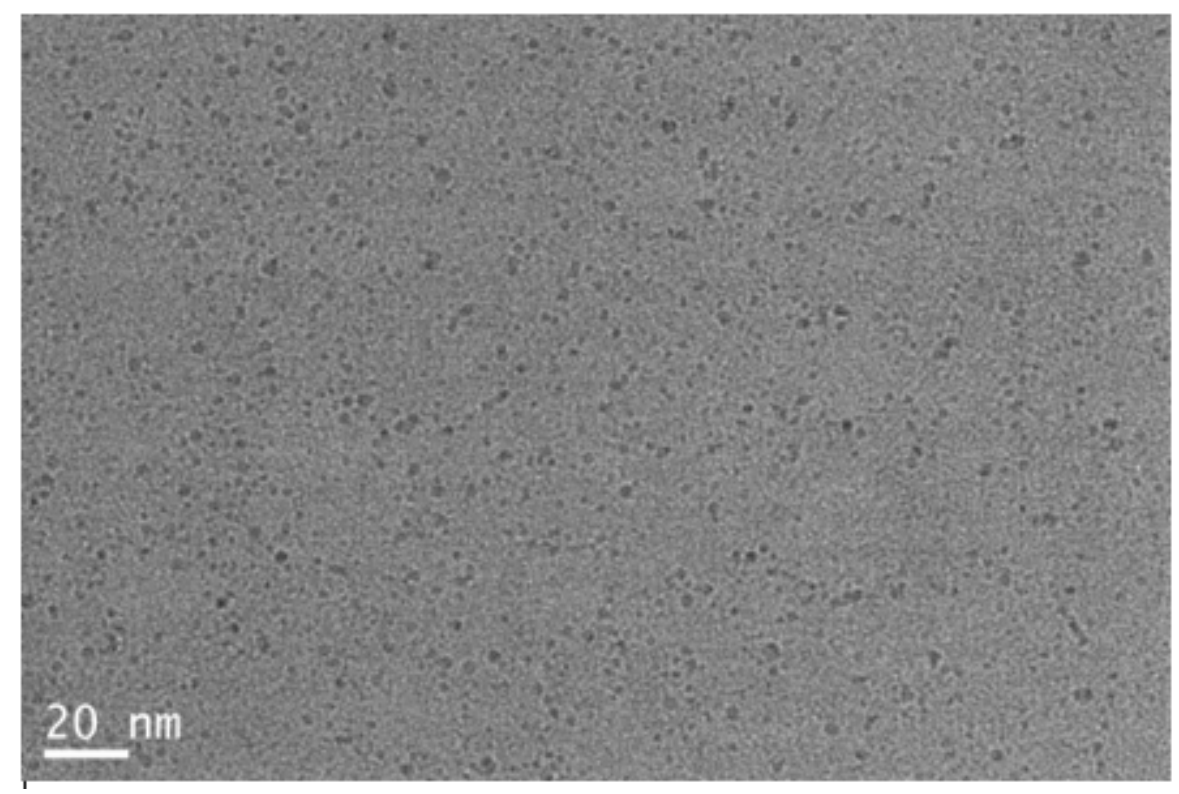

\section{Figure 1}

TEM image of CDs 
(a)

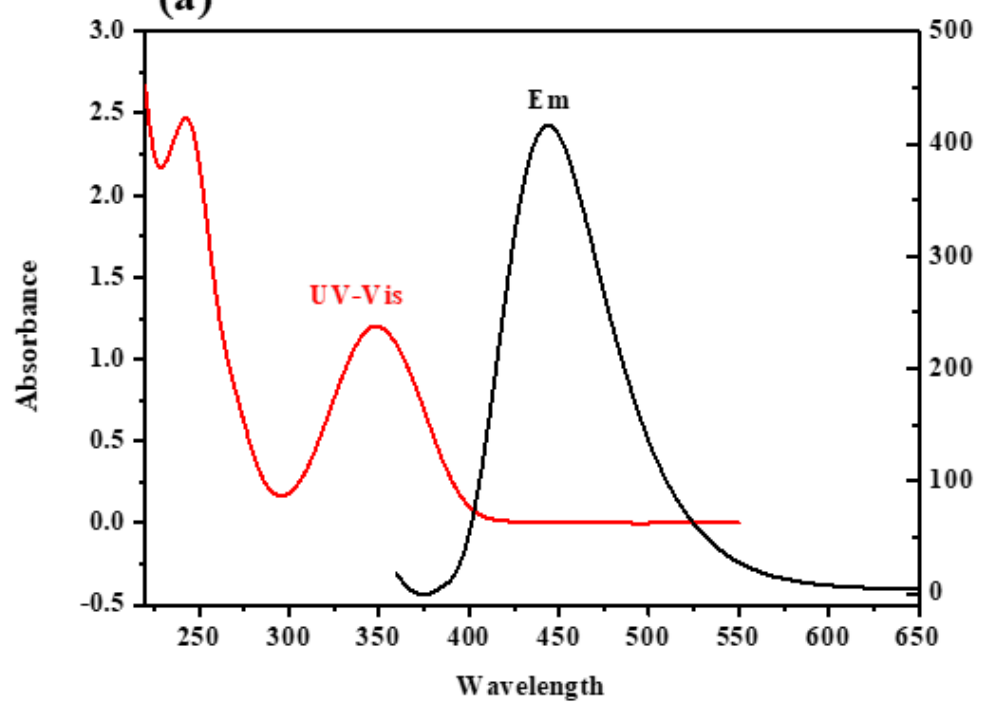

(b)

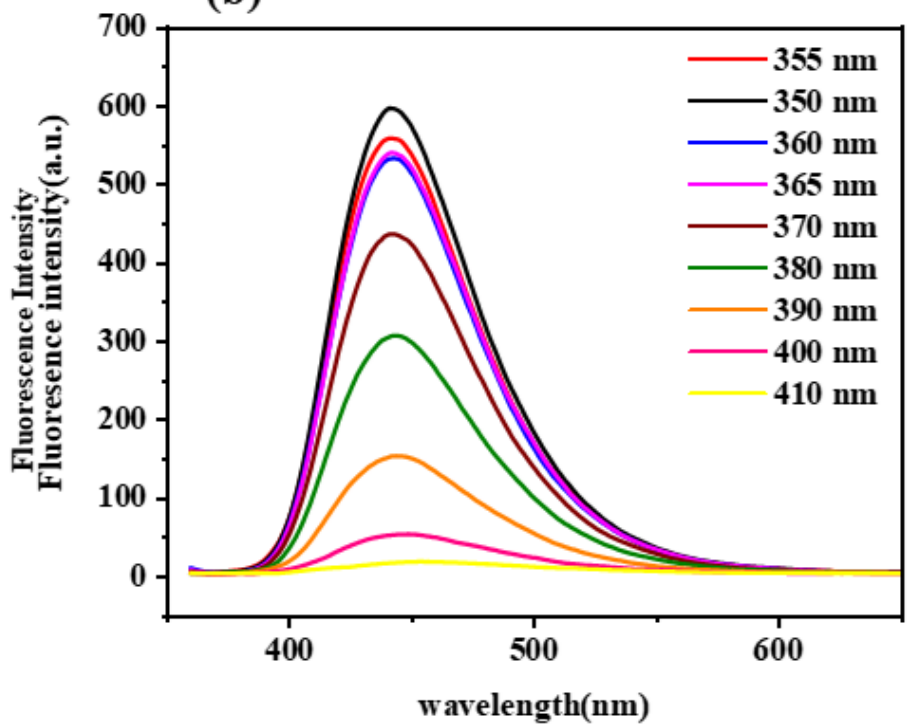

Figure 2

a UV-vis spectra and fluoresence emission spectra of CDs囚b Fluorescence spectra of the CDs at different excitation wavelengths.

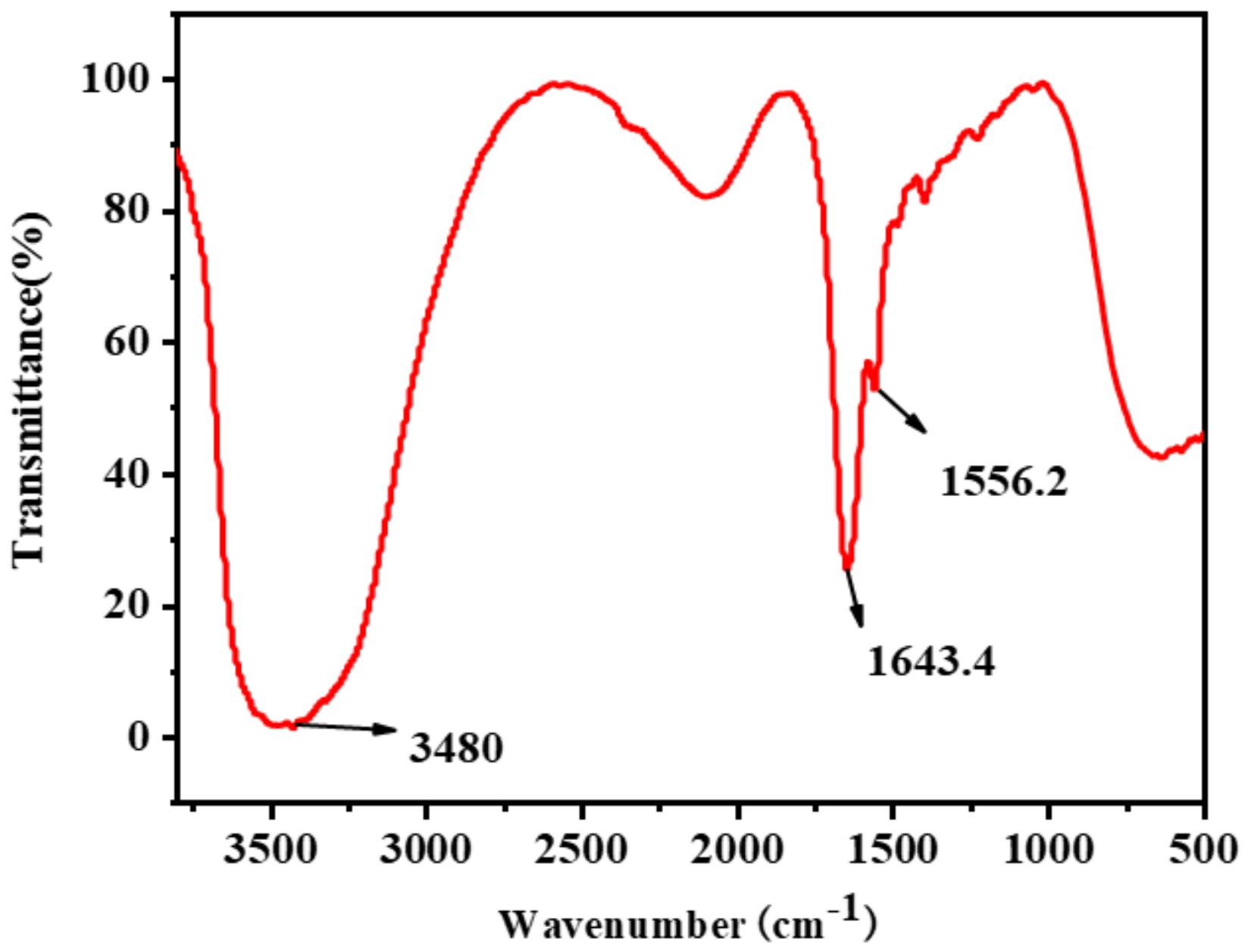


Figure 3

FT-IR of the CDs

(a)

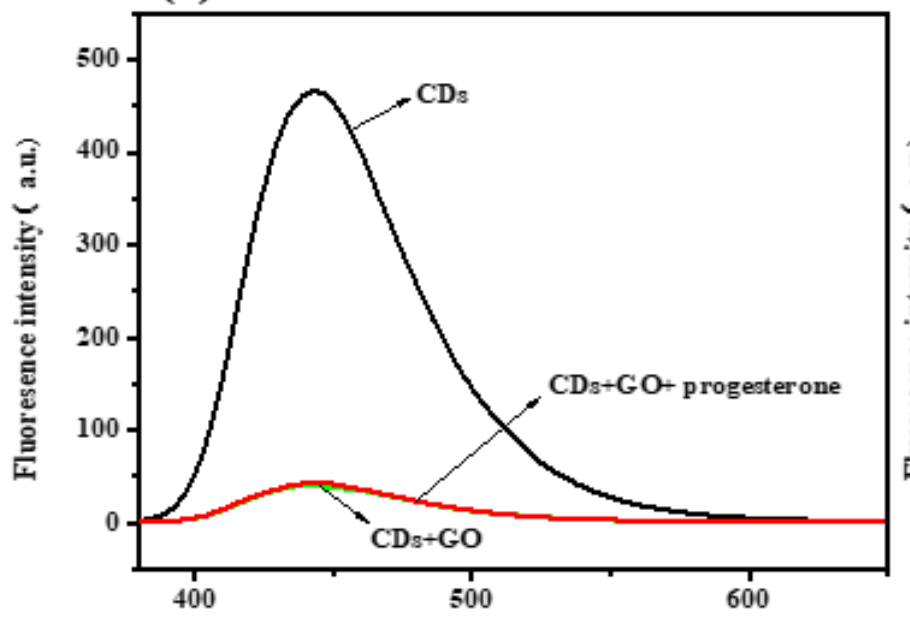

(c) Wavelength(nm)

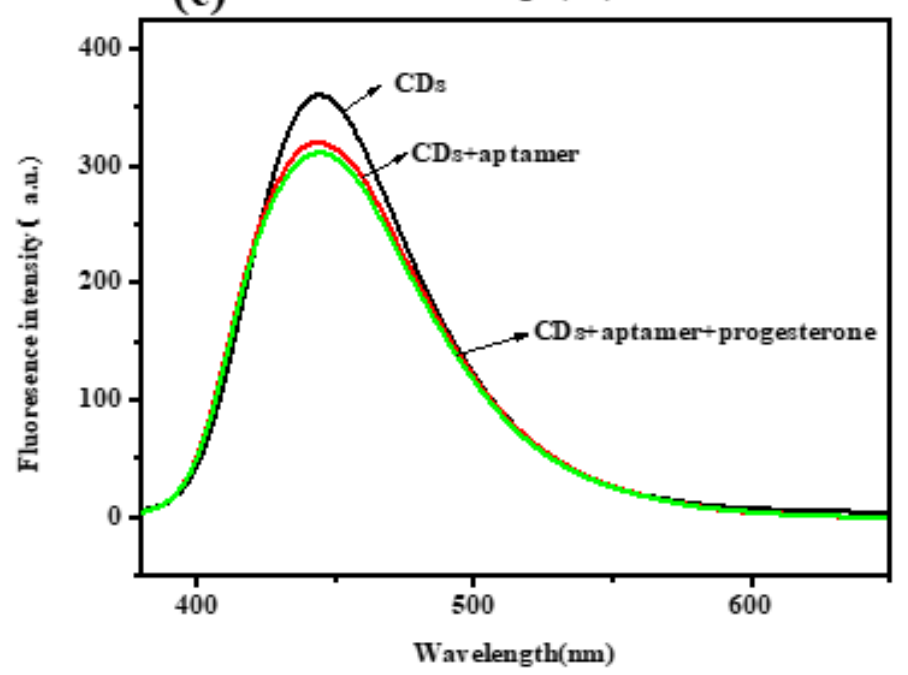

(b)

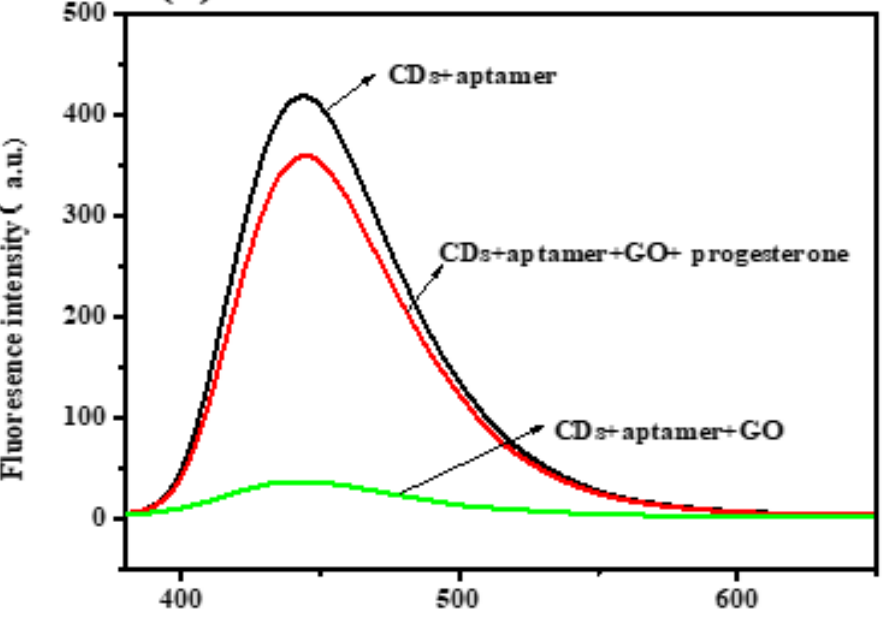

(d)

Wavelength(nm)

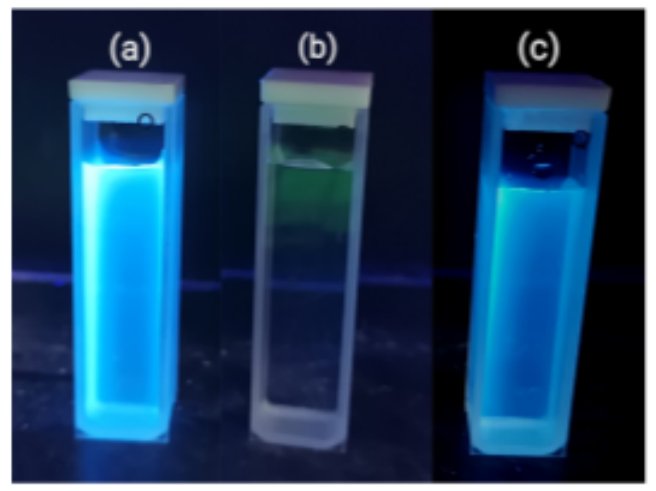

\section{Figure 4}

a Fluorescence emission spectra of $C D s \square C D s+G O$ and $C D s+G 0+P 4 \rrbracket b$ Fluorescence emission spectra of CDs+aptamer $\square C D s+a p t a m e r+G O$ and $C D s+a p t a m e r+G O+P 4 \llbracket c$ Fluorescence emission spectra of CDs $\square$ CDstaptamer and CDstaptamer+P4『d The color change of (a) CDs-aptamer (b) CDs-aptamer-GO (c) CDs-aptamer-GO-P4 under UV light. 

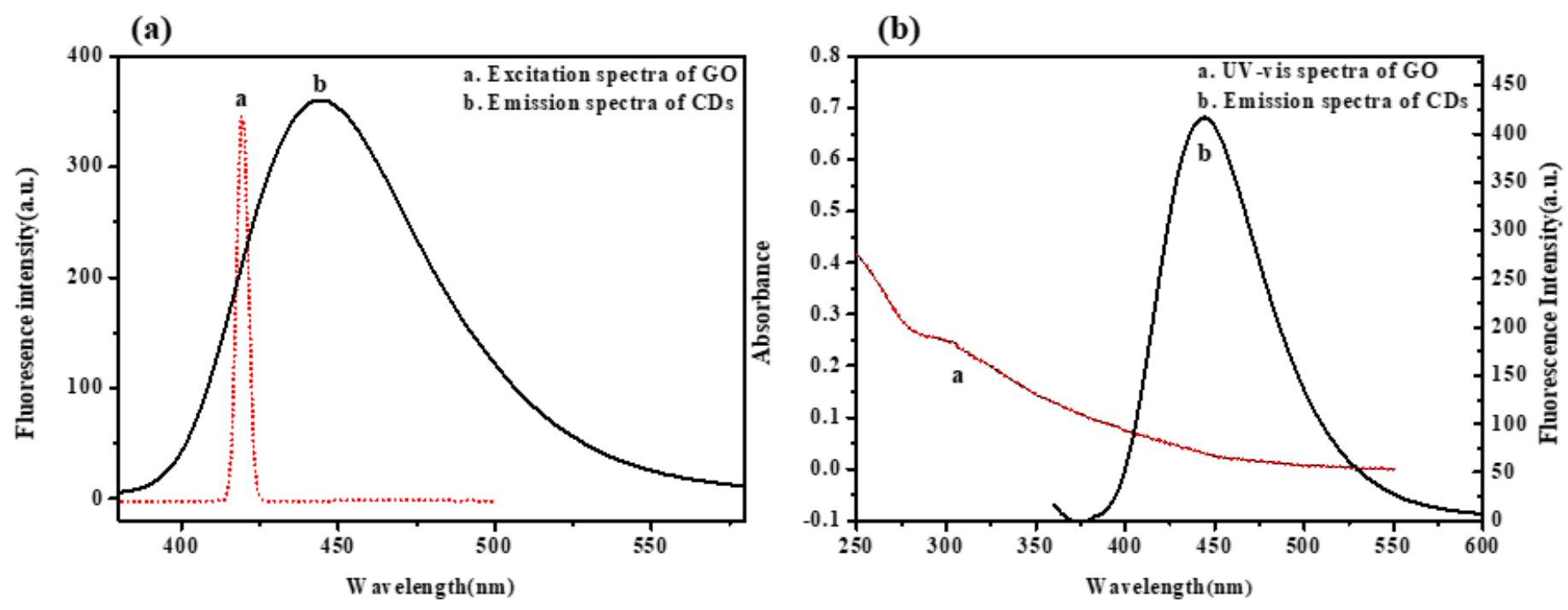

Figure 5

a Excitation spectra of GO and emission spectra of CDs $\bigotimes b$ UV-vis spectra of GO and emission spectra of CDs. 
(a)

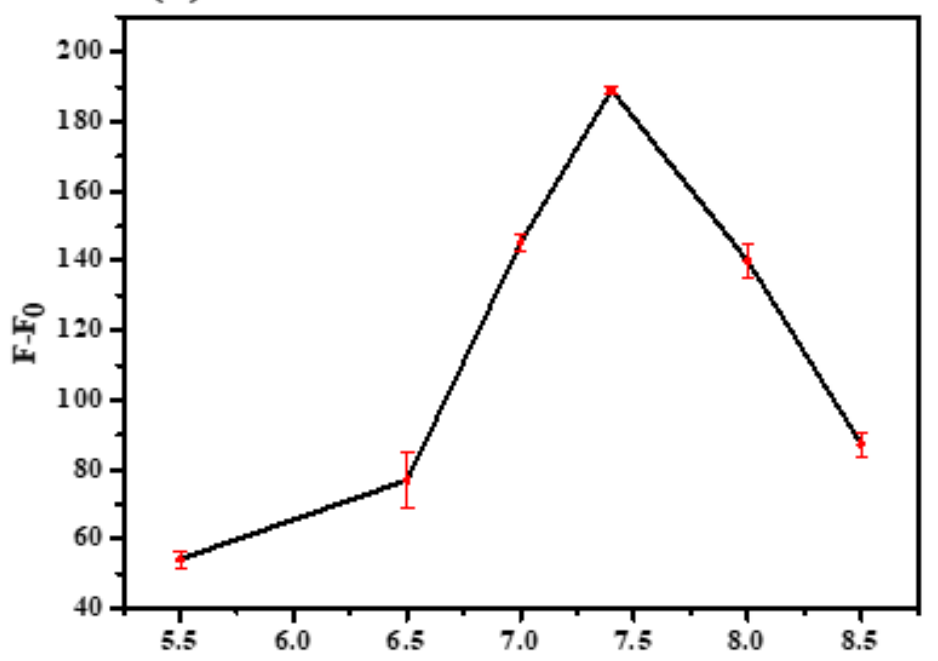

(c)

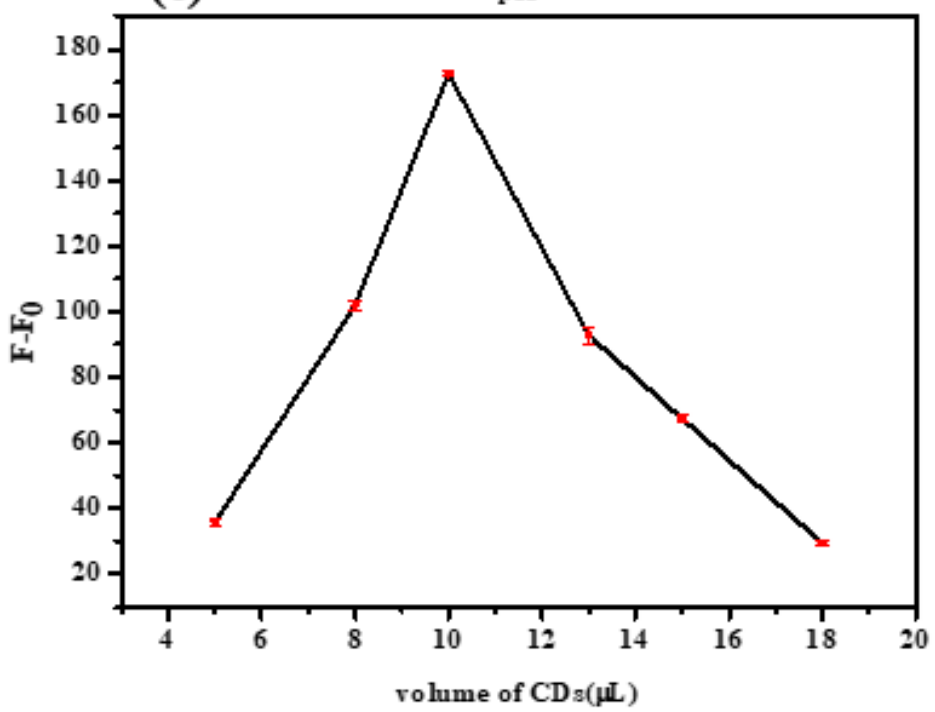

(b)

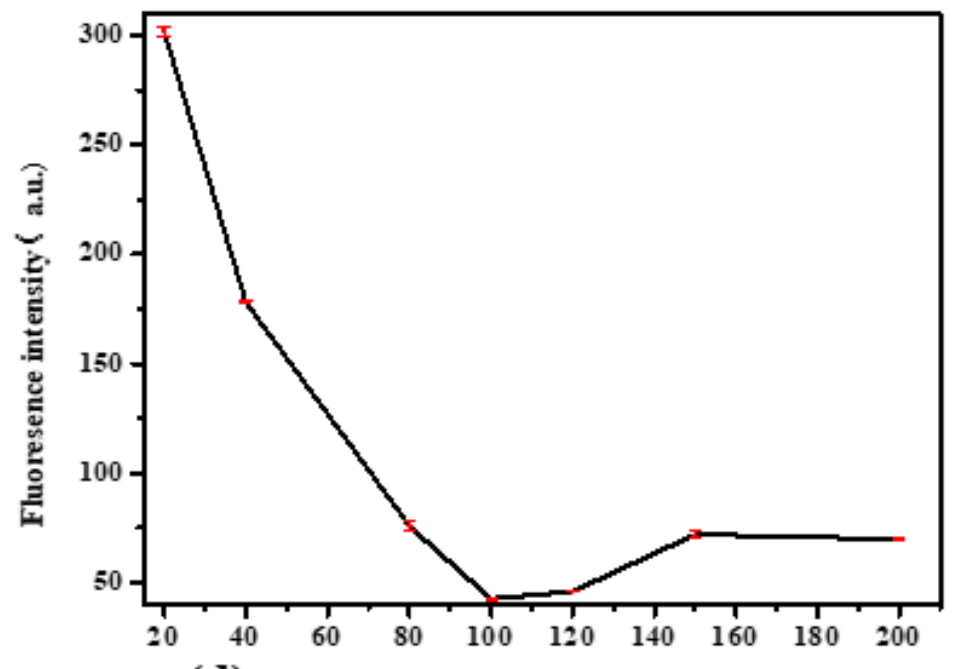

(d)

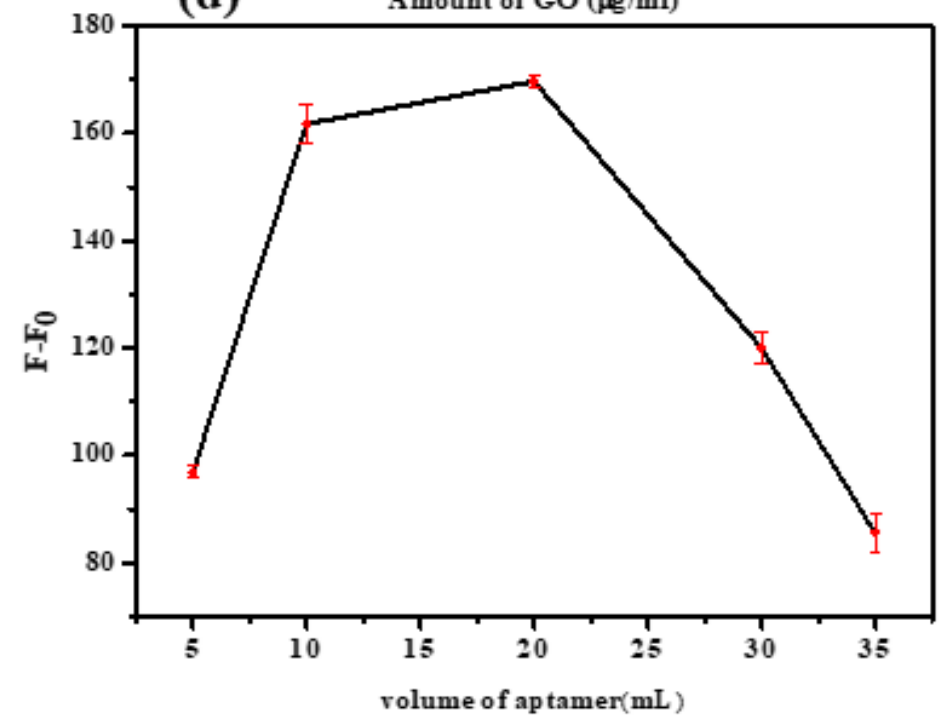

Figure 6

a the influence of $\mathrm{pH}$ on the fluorescence intensity difference value (F-F0) $\mathbb{b}$ the influence of the amount of GO on the fluorescence intensity FO囚c the influence of the volume of CDs on the fluorescence intensity difference value ( $F-F 0) \llbracket d$ the influence of the volume of aptamer on the fluorescence intensity difference value (F-F0). 
(a)

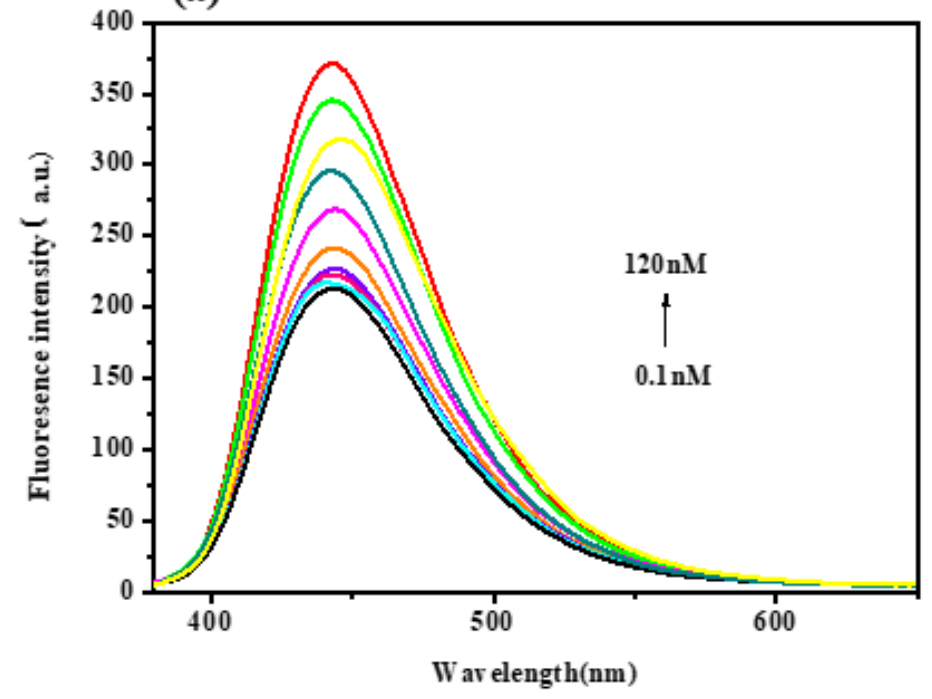

(b)

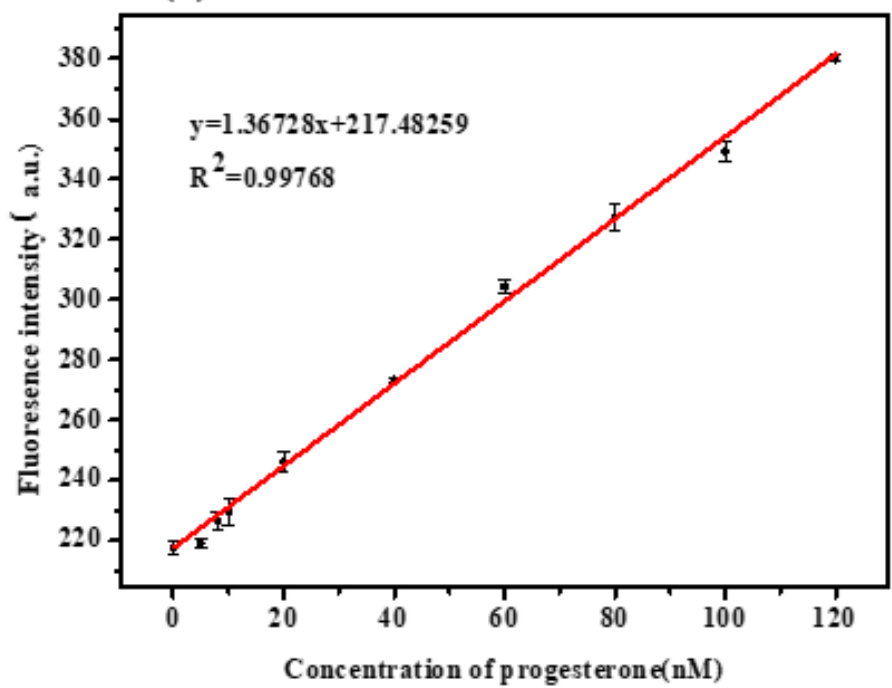

Figure 7

a Emission spectrum of the CDs-aptamer-GO in the addition of different concentration of progesterone from $0.1 \mathrm{nM}$ to $120 \mathrm{nM}(0.1,5,8,10,20,40,60,80,100$ and $120 \mathrm{nM}) \otimes \mathrm{b}$ linear calibration curve of the aptasensor for progesterone.

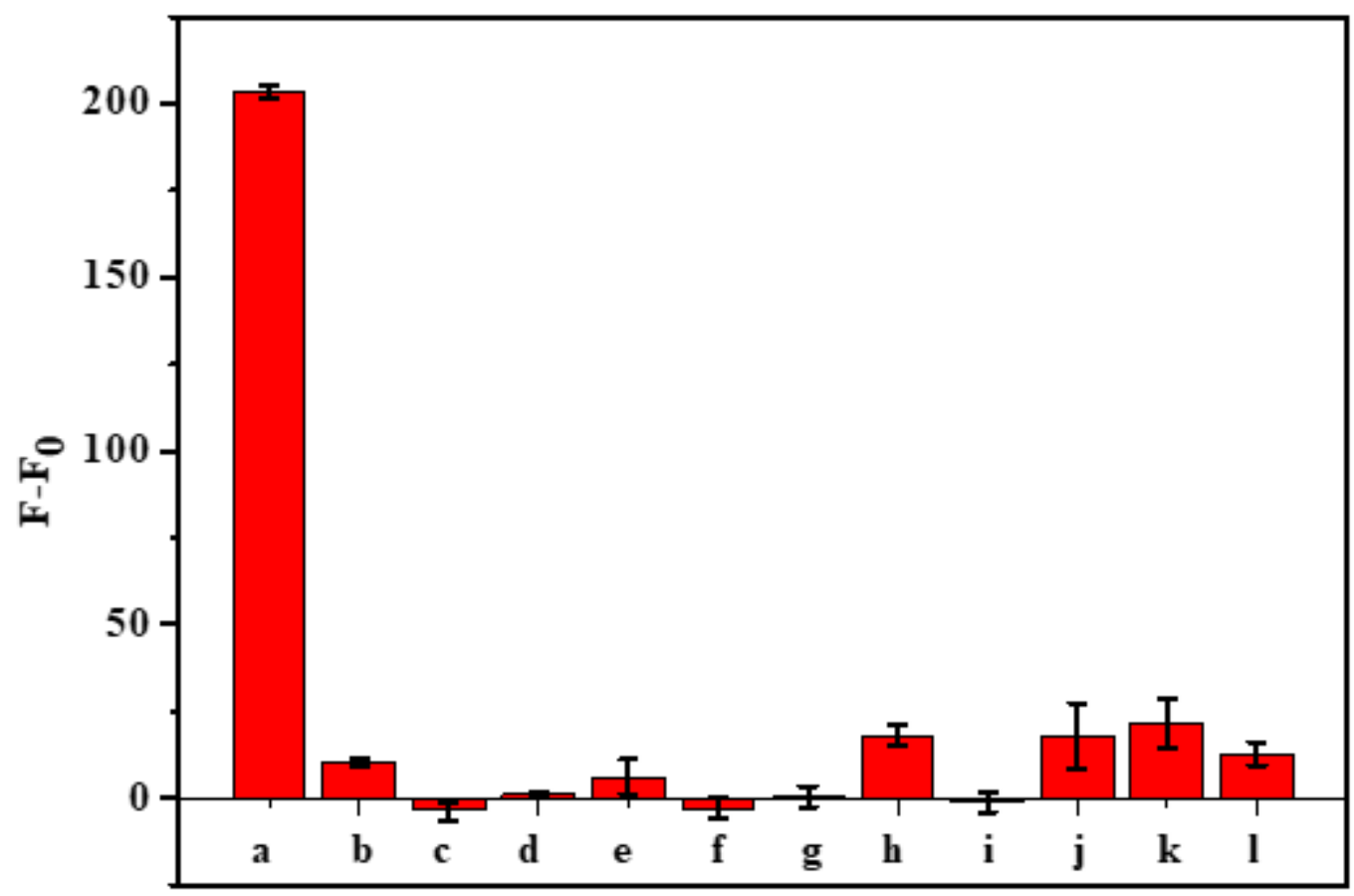

Figure 8

Selective determination of $120 \mathrm{nM}$ progesterone and $3 \times 120 \mathrm{nM}$ other potential interfering substances. (Other potential interfering substances include (a) progesterone, (b) L-cysteine, (c) L-adrenaline, (d) 
ascorbic acid, (e) glutathione, (f) $\mathrm{KCl},(\mathrm{g}) \mathrm{NaCl}$, (h) $\mathrm{MnCl}$ 2, (i) $\mathrm{CdCl}$ 2, (j) dopamine, (k) estrone, (l) estradiol.) 